\title{
L1-NORM FITTING OF ELLIPTIC PARABOLOIDS WITH PRIOR INFORMATION FOR ENHANCED CONIFEROUS TREE LOCALIZATION IN ALS POINT CLOUDS
}

\author{
P. Polewski ${ }^{1}$, W.Yao ${ }^{1,2, *}$ M. Heurich ${ }^{3,4}$ \\ ${ }^{1}$ Dept. of Land Surveying and Geo-Informatics, The Hong Kong Polytechnic University, Hung Hom, Hong Kong - \\ \{przemyslaw.polewski,wei.hn.yao\}@ polyu.edu.hk \\ ${ }^{2}$ Research Institute for Sustainable Urban Development, The Hong Kong Polytechnic University, Hung Hom, Hong Kong \\ ${ }^{3}$ Dept. for Conservation and Research, Bavarian Forest National Park, 94481 Grafenau, Germany - marco.heurich@npv-bw.bayern.de \\ ${ }^{4}$ Chair of Wildlife Ecology and Management, University of Freiburg, 79106 Freiburg, Germany
}

KEY WORDS: 3D shape fitting, quadratic programming, precision forestry, QCQP

\begin{abstract}
:
Airborne laser scanning (ALS) is an established tool for deriving various tree characteristics in forests. In some applications, an accurate pointwise estimate of the tree position is required. For dense data acquired by TLS or UAV-mounted scanners, this can be achieved by locating the stem, whose center coordinates are then used for deriving the planimetric tree position. However, in case of standard ALS data this is often not an option due to the low probability of obtaining stem hits in operational scenarios of forest mapping campaigns. This paper presents an alternative, indirect approach where the tree position is approximated as the center of a quadric surface which best represents the tree crown shape. The study targets coniferous trees due to their distinct crown shape which may be approximated by an elliptic paraboloid. It is assumed that individual tree point clusters are given and the task is to find the tree center for each cluster. We first consider the general problem of fitting an elliptic paraboloid with a known axis and an L1 residual norm error criterion, which is more robust to outliers compared to least-squares fitting. We formulate this problem as a quadratically constrained quadratic program (QCQP), and show how prior knowledge on the crown shape and center position can be incorporated. Next, a computationally simpler problem is considered where the paraboloid semiaxis lengths are constrained to be equal, and a corresponding linear program is constructed. Experiments on ALS datasets of forest plots from Bavaria, Germany and Oregon, USA reveal that a reduction in median tree position error of up to $20 \%$ can be attained compared to both least-squares fitting and other baseline techniques, resulting in an absolute error of ca. $22 \mathrm{~cm}$ on both datasets.
\end{abstract}

\section{INTRODUCTION}

Airborne laser scanning (ALS) has become an increasingly common technique for estimating parameters of both entire stands and single trees in forested areas. In the latter case, single-tree approaches attempt to first segment individual trees within the point cloud and then derive parameters of interest for each found tree (Reitberger et al., 2009). One such parameter is the tree's planimetric location, which can be though of as the center position of the tree's stem at ground level. A common use of the extracted tree locations is associated with evaluating the quality of a tree segmentation algorithm, where a pointwise estimate of the detected tree position is necessary for comparison with a reference location. Another important application of the tree positions is for accurate co-registration of multi-modal aerial and terrestrial LiDAR point clouds/images of forest scenes based on pairwise distances between trees within the scene (Polewski et al., 2019; Lee et al., 2016). Also, from a practical perspective, precise information about tree positions is crucial for the operation of autonomous logging machines (Hellström, 2008), which navigate through the forest and automatically harvest designated trees. Finally, the emerging technology for geo-location and navigation of autonomous vehicles in GPS-denied areas often relies on accurate tree positions as a robust terrain feature for orientation (Hussein et al., 2015). The matching process between found tree positions via parameter optimization of an appropriate transform can ensure an alignment with a georeferenced coordinate system.

\footnotetext{
${ }^{*}$ Corresponding author
}

When the point density is sufficiently high, direct approaches to detecting stems can be applied, such as in case of TLS data (Liang et al., 2012) or high-density ALS based on low-altitude helicopter flights (Amiri et al., 2017). On the other hand, while stem detection in ordinary ALS data has been attempted (Reitberger et al., 2007), generally it is not realistic to expect that laser reflections of stems will be available for most trees. Therefore, heuristic or indirect methods for deriving tree locations have to be applied. A common method of tree position detection relies on finding tree tops using local maxima filtering of the canopy height model (CHM). However, in some cases such a procedure can lead to significant deviations between the detected and true tree positions. The lack of robustness can be attributed to the fact that this method only relies on one laser reflection (having the highest $\mathrm{Z}$ coordinate), so a single outlier can lead to failure. Another popular approach assumes that the tree center coincides with the planimetric centroid of the 3D point cluster which represents the entire tree (Yao et al., 2013). However, in practice the segmented tree clusters are seldom perfect, biasing the centroid away from the true tree position. In general, the main drawback of such heuristic methods is that they do not take into account the 3D shape of the segmented trees, which has been well-studied and is amenable to modeling with geometric surfaces. In particular, in environmental science literature the crown surface of coniferous trees is often modeled as a paraboloid (Husch et al., 2002).

In this paper, we make use of this intuition about the tree crown shape, and introduce an alternative approach to tree position calculation, based on fitting elliptic paraboloids to point 
clouds representing individual trees. Our primary contribution is the quadratic programming formulation of fitting elliptic paraboloids having a known axis, according to the $L_{1}$-norm of algebraic distances, with the ability to incorporate prior information about the shape and position of the quadric surface. The known axis assumption represents the apriori knowledge that most tree stems are approximately parallel to the world $\mathrm{Z}$ coordinate axis due to gravitropism. This assumption yields a significant simplification of the general unconstrained paraboloid fitting problem, although in reality the planimetric tree top and stem ground positions may be shifted by a certain offset due to crown asymmetry or a non-negligible deviation of the stem from the $\mathrm{Z}$ axis. Although the models and algorithms are introduced in the context of precision forestry, they are generic and could be re-used for similar paraboloid fitting problems, e.g. industrial inspection/modeling of manufactured parts.

The rest of this paper is structured as follows. Section 2 deals with previous applications of paraboloids to tree crown modeling, as well as related problems of quadric surface fitting which inspired this work. In Section 3, the mathematical formulation of the elliptic paraboloid fitting problem is given. The next section describes how prior knowledge on the shape (semiaxis length relationships) as well as position can be incorporated into the basic problem. The experimental setup, used evaluation metrics as well as data acquisition campaigns are described in Section 5, followed by presentation and discussion of results (Sec. 6). Finally, the most important conclusions from this study are stated in Section 7.

\section{RELATED WORK}

The idea to apply $L_{1}$ techniques to paraboloid fitting is motivated by the work of (Zhou, Salvado, 2011), who showed that $L_{1}$ fitting of ellipsoids provides a significant advantage over the $L_{2}$ norm for pose normalization of hippocampal shapes. The conceptually closest approach to ours is due to Xiao et al. (2016), which proposes to find the tree center through fitting an ellipsoid to the upper part of the crown, according to the modified version of the Pollock model (Pollock, 1994). Similar to our method, a rotation angle in the XY plane is included in the model. However, the fitting is performed in the least-squares $\left(L_{2}\right)$ sense compared to the $L_{1}$-norm used in our study, and no prior knowledge is introduced into the optimization problem. Also, the authors did not investigate the quality of the obtained tree positions as the study was focused on other parameters. Quadric surfaces in forestry remote sensing have mostly been applied for deriving features relevant to tree species classification, such as mean and Gaussian curvature (Barilotti et al., 2009). Reitberger et al. (2008) perform $L 2$ paraboloid fitting to tree crown points and use the obtained semiaxis lengths as features for distinguishing between coniferous and deciduous species. Recently, Amiri et al. (2018) showed that by embedding an explicit modeling step of coniferous tree crowns with paraboloids into a graph-cut segmentation procedure, it is possible to significantly improve the accuracy of the segmented individual trees. The task of fitting elliptic paraboloids has been extensively addressed in literature as part of the broader problem of fitting quadrics. Dai et al. (2007) present a method for recovering the elliptic paraboloid parameters from least-squares fitting of generic quadric surfaces. Al-Subaihi (Watson) consider the problem of quadric fitting with respect to $L_{1}$ and $L_{\infty}$. In general, existing methods for $L_{1}$ optimization do not allow direct incorporation of prior knowledge on the paraboloid's shape. Also, since the problem is non-convex, the algorithms may converge to a local minimum only. We propose to formulate the fitting problem as a quadratic program, which both admits prior information and provides a systematic approach for exploring the solution space, often leading to global optimality. Indeed, although the resulting quadratic program is non-convex and hence NP-hard, in practice a provably optimal solution can usually be obtained using branch-and-bound techniques in reasonable time.

\section{FITTING ELLIPTIC PARABOLOIDS}

\subsection{Quadric surface parametrization}

An elliptic paraboloid surface in standard position (centered at the origin, with the $Z$ axis as its axis) can be described by the following equation (Dai et al., 2007):

$$
\hat{x}^{T} A_{0} \hat{x}+g \hat{x}=0
$$

where $\hat{x}=[x, y, z]$ represents the point coordinates and $g=$ $[0,0,-1]$. Let $a, b$ indicate the paraboloid's semiaxis lengths. Then, for a 'downward' paraboloid, $A_{0}$ is a diagonal matrix with the diagonal $\left[-1 / a^{2},-1 / b^{2}, 0\right]$. We consider a paraboloid with its center located at $\left[x_{0}, y_{0}, z_{0}\right]$, parallel to the $Z$ axis and rotated around $Z$ by an angle of $\theta$. Letting $R$ be the standard rotation matrix around the $Z$ axis by $\theta$, and setting $\bar{x}=[x-$ $\left.x_{0}, y-y_{0}, z-z_{0}\right], A=R^{T} A_{0} R$ we can write the rotated paraboloid as:

$$
z=\bar{x}^{T} A \bar{x}+z_{0}
$$

Let $S=\left\{p_{1}, \ldots, p_{n}\right\}, p_{i}=\left[x_{i}, y_{i}, z_{i}\right]$ be a set of $n$ measured points representing the paraboloid surface. The above equation could be used directly to optimize the algebraic distances between the modeled and measured heights on $S$ with respect to the 6 paraboloid parameters $\Theta=\left[a, b, \theta, x_{0}, y_{0}, z_{0}\right]$ and a chosen residual norm $L_{p}$, with per-point weights $W$ :

$$
\begin{gathered}
\min _{\Theta}\|W r\|_{p} \\
r=\left[z_{1}-z\left(x_{1}, y_{1} ; \Theta\right), \ldots, z_{n}-z\left(x_{n}, y_{n} ; \Theta\right)\right]
\end{gathered}
$$

However, the problem is strongly non-linear in the original paraboloid parameters $\Theta$, and therefore it is likely that any stationary point-finding method will terminate in a local optimum. Moreover, since we are interested in finding the solution for $p=1$, i.e. the $L_{1}$ norm, any gradient-based method could be faced with non-differentiability problems whenever one of the residuals $r_{i}$ becomes exactly 0 . To alleviate such difficulties, we may resort to an alternative parametrization $\Phi$ where $z(x, y ; \Phi)$ is a linear function of the parameters (Dai et al., 2007; Hall et al., 1982):

$$
z(x, y ; \Phi)=\phi_{0} x^{2}+\phi_{1} y^{2}+\phi_{2} x y+\phi_{3} x+\phi_{4} y+\phi_{5}
$$

Note that due to the known paraboloid axis, there are 6 parameters compared to 9 for the unconstrained quadric (see Fig. 1). The problem $\min _{\Phi}\|W r\|_{p}$ can now be written more explicitly in matrix form as $\min _{\Phi}\|W(D \Phi-\bar{z})\|_{p}$, where $\bar{z}=\left[z_{1}, \ldots, z_{n}\right]$ is the vector of measured heights, and the $i$ th row of $D$ is defined as $D_{i}=\left[x_{i}^{2}, y_{i}^{2}, x_{i} y_{i}, x_{i}, y_{i}, 1\right]$.Taking the $L_{2}$ norm, we obtain the familiar least-squares problem, for which a closed-form optimal solution exists:

$$
\tilde{\Phi}_{L_{2}}=\left(D^{T} W D\right)^{-1} D^{T} W \bar{z}
$$


Here, we are concerned with the $L_{1}$ solution, motivated by the robustness of $L_{1}$ optimization methods in the presence of outliers, and in particular the successful application to ellipsoid fitting (Zhou, Salvado, 2011). It is well known that $L_{1}$ minimization problems can be solved using linear programming. Our problem for $p=1$ can then be formulated as a linear program, with the introduction of auxiliary variables $\Gamma=\left[\gamma_{1}, \ldots, \gamma_{n}\right]$ :

$$
\begin{aligned}
& \min _{\Phi, \Gamma} \sum_{i=1}^{n} W_{i i} \gamma_{i} \\
& \left.\begin{array}{rl} 
& D_{i} \Phi^{T}-z_{i} \leq \gamma_{i} \\
\text { s.t. } & -D_{i} \Phi^{T}+z_{i} \leq \gamma_{i}
\end{array}\right\} \forall_{i \in 1, \ldots, n}
\end{aligned}
$$

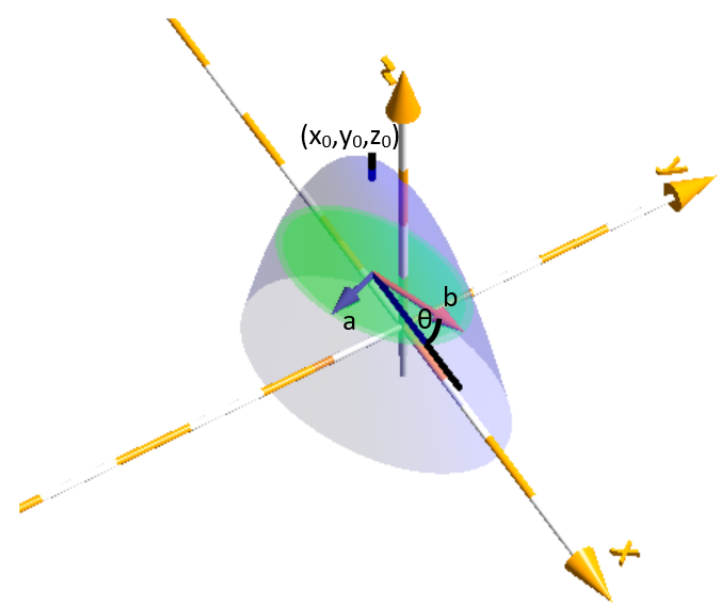

Figure 1. Z-axis aligned elliptic paraboloid centered at $\left(x_{0}, y_{0}, z_{0}\right)$, rotated by $\theta$ degrees around the $\mathrm{Z}$ axis, with semiaxis lengths $a, b$.

\subsection{Elliptic paraboloid constraints}

While Eq. 4 may describe an elliptic paraboloid, this will not necessarily be true for any combination of $\Phi$ coefficient values. Indeed, other quadric surface types such as a hyperbolic paraboloid, cylinder or plane are also representable in this parametrization. The concrete shape type obtained from fitting with Eq. 6 is data-dependent and cannot be pre-specified without additional constraints. While this is less of a concern for highly accurate point clouds obtained e.g. from close-range scanning, in case of ALS point clouds this problem can manifest itself. Particularly when fitting incomplete point clouds of tree crowns, distorted by imperfect segmentation techniques, an explicit constraint on the desired surface type (elliptic paraboloid) is required. To derive it, we consider the two quadric surface invariants $D$ and $\Delta$ (Dai et al., 2007), which together determine the surface type. The surface is an elliptic paraboloid iff $D=0$ and $\Delta<0$. Whereas the former condition is fulfilled automatically due to the formulation of Eq. 4, the latter one must be addressed directly. The invariant $\Delta$ can be expressed as a function of the surface coefficients in the form: $\Delta=\frac{1}{4}\left(\frac{\phi_{2}^{2}}{4}-\phi_{0} \phi_{1}\right)$. Consequently, the quadratic constraint (C2) must be added to problem (6) to ensure that an elliptic paraboloid is obtained:

$$
\frac{\phi_{2}^{2}}{4}-\phi_{0} \phi_{1}<0
$$

Finally, to ensure that the paraboloid opens 'downward' along the $Z$ axis, an upper bound $\phi_{0}, \phi_{1}<0$ is required. The addition of $(\mathrm{C} 2)$ turns the linear program into a non-convex quadratically constrained quadratic program (QCQP), which can be approximately solved using the standard spatial branch-and-bound technique (Belotti et al., 2009).

\subsection{Recovering shape parameters}

Once the generic surface parameters $\Phi$ are obtained through optimization, it is still necessary to recover the shape parameters specific to elliptic paraboloids, i.e. the original vector $\Theta$. This can be achieved by expanding Eq. 2 and grouping coefficients associated with appropriate products of coordinates $x, y$, resulting in a formulation similar to Eq. 4 . Comparing corresponding coefficients yields the relationship between $\Phi$ and $\Theta$. Let $A_{1}, b_{1}$ be matrix functions of $\Phi$ such that:

$$
A_{1}=\left(\begin{array}{cc}
\phi_{0} & \frac{\phi_{2}}{2} \\
\frac{\phi_{2}}{2} & \phi_{1}
\end{array}\right), b_{1}=\left(\begin{array}{l}
\phi_{3} \\
\phi_{4}
\end{array}\right)
$$

Then, the center $\left(x_{0}, y_{0}, z_{0}\right)$ of the paraboloid can be found as the solution of the following linear system:

$$
\begin{gathered}
\left(\begin{array}{l}
x_{0} \\
y_{0}
\end{array}\right)=\left(-2 A_{1}\right)^{-1} b_{1} \\
z_{0}=\phi_{5}-\left(\phi_{0} x_{0}^{2}+\phi_{1} y_{0}^{2}+\phi_{2} x_{0} y_{0}\right)
\end{gathered}
$$

The semiaxis lengths $a, b$ can be obtained through eigendecomposition of $A_{1}$ (Dai et al., 2007). Let $\lambda_{1}, \lambda_{2}$ be the two eigenvalues of $A_{1}$ and let $\nu$ be the eigenvector associated with $\lambda_{1}$, then:

$$
a=\frac{1}{\sqrt{-\lambda_{1}}}, b=\frac{1}{\sqrt{-\lambda_{2}}}, \theta=\operatorname{atan}\left(\nu_{y}, \nu_{x}\right)
$$

Note that enforcing the constraints from Sec. 3.2 guarantees the negativity of $\lambda_{1}, \lambda_{2}$ as well as the invertibility of $A_{1}$, so that a unique solution $\left(x_{0}, y_{0}, z_{0}\right)$ exists.

\section{INTRODUCING PRIOR KNOWLEDGE}

In this section, we describe how two types of prior knowledge about the paraboloid's shape and position can be incorporated into the optimization problem. Also, the final formulations of the quadratic and linear programs are presented.

\subsection{Semiaxis relationships}

4.1.1 Ratio of semiaxis lengths Oftentimes, there is some prior knowledge on the desired degree of imbalance/asymmetry of the paraboloid's semiaxes. In particular, tree crowns tend to be quite symmetrical. Ideally, we would like to enforce bounds on the ratio $r_{0}=a /(a+b)$. Unfortunately, the closed-form expression for $r_{0}$ in terms of the program's variables $\Phi$ is quite complicated and would introduce more non-convexity into the QCQP. Instead, we target $r_{1}=a^{2} /\left(a^{2}+b^{2}\right)$ and show how this ratio can be bounded by adding a single constraint on $\phi_{0}, \phi_{1}, \phi_{2}$. Consider once more Eq. 2 expanded to the explicit form of Eq. 4. The coefficients next to $x^{2}, y^{2}$ are respectively $A_{11}, A_{22}$, therefore it must hold that $\phi_{0}+\phi_{1}=$ $A_{11}+A_{22}$. Examining the difference $\phi_{1}-\phi_{0}$ and noting that $\phi_{2}=A_{12}+A_{21}$, after expanding $A=R^{T} A_{0} R$ and applying 
basic algebraic transformations we obtain:

$$
\begin{gathered}
\left(\phi_{0}+\phi_{1}\right)^{2}=\left(\frac{1}{a^{2}}+\frac{1}{b^{2}}\right)^{2}\left[\sin ^{2} \theta+\cos ^{2} \theta\right]^{2} \\
\left(\phi_{0}-\phi_{1}\right)^{2}+\phi_{2}^{2}=\left(\frac{1}{b^{2}}-\frac{1}{a^{2}}\right)^{2}\left[\sin ^{2} 2 \theta+\cos ^{2} 2 \theta\right]
\end{gathered}
$$

Since the right-hand side trigonometric expressions evaluate to one, this yields expressions for the sum and difference of squared semiaxis lengths. These expressions can in turn be used for computing the ratio $r_{1}$ :

$$
\rho=\frac{1 / b^{2}-1 / a^{2}}{1 / b^{2}+1 / a^{2}}=1-2 r_{1}
$$

Indeed, a constraint of the form $\rho^{2} \leq \omega^{2}$ implies $r_{1} \in$ $\left[\frac{1-\omega}{2} ; \frac{1+\omega}{2}\right]$ for $0 \leq \omega \leq 1$. This constraint can be written as $\left(\left(\phi_{0}-\phi_{1}\right)^{2}+\phi_{2}^{2}\right) /\left(\phi_{0}+\phi_{1}\right)^{2} \leq \omega^{2}$, or more explicitly:

$$
\left(1-\omega^{2}\right) \phi_{0}^{2}+\left(1-\omega^{2}\right) \phi_{1}^{2}+\phi_{2}^{2}+\left(-2-2 \omega^{2}\right) \phi_{0} \phi_{1} \leq 0
$$

Similar to $(\mathrm{C} 2),(\mathrm{C} 3)$ is also non-convex due to the bilinear term $\phi_{0} \phi_{1}$, however this bilinear term is also shared by both constraints, which can be exploited by the branch-and-bound algorithm during solving.

4.1.2 Semiaxis length upper bound It would be useful to introduce simple upper bounds on the semiaxis lengths, reflecting knowledge about e.g. the maximum crown diameter of certain tree species. Unfortunately, a simple expression for $a, b$ in terms of the program's variables $\phi_{i}$ is not available. However, using techniques similar to the ones described in the previous paragraph, an upper bound constraint may be derived. Using the building blocks from Eq. 11, we may write:

$$
\left(\frac{1}{a^{2}}+\frac{1}{b^{2}}\right)^{2}-\left(\frac{1}{b^{2}}-\frac{1}{a^{2}}\right)^{2}=\frac{4}{a^{2} b^{2}}
$$

Consider the constraint $4 /\left(a^{2} b^{2}\right) \geq \mu^{2}$, which may be equivalently expressed as $a^{2} b^{2} \leq 4 / \mu^{2}$, and assume without loss of generality that $b \geq a$. If constraint (C3) is fulfilled, from Eq. 12 we have that $a^{2} \geq b^{2}(1-\omega) /(1+\omega)$. Putting the two inequalities together, we obtain an upper bound for $b^{4}$ :

$$
b^{4} \leq \frac{4}{\mu^{2}} \frac{1+\omega}{1-\omega}
$$

The constraint $4 /\left(a^{2} b^{2}\right) \geq \mu^{2}$ needs to be formulated in terms of the variables $\phi$. This can be achieved by applying the expressions from Eqs. 11,14, leading to the final form:

$$
\phi_{2}^{2}-4 \phi_{0} \phi_{1} \leq-\mu^{2}
$$

This inequality is surprisingly similar to constraint (C2), Eq. 7. Indeed, dividing both sides by 4 , the exact form of (C2)'s left-hand side is recovered: $\phi_{2}^{2} / 4-\phi_{0} \phi_{1} \leq-\mu^{2} / 4$. Because $\mu^{2}$ is necessarily positive (to avoid division by zero in Eq. 15), any parameter vector $\Phi$ which fulfills (C4) must also automatically fulfill (C2), making the latter constraint redundant. By enforcing (C4) with an appropriate constant $\mu^{2}$, an arbitrary upper bound on the maximal semiaxis length can be obtained. However, it should be noted that this bound is qualitatively different from two independent constraints $a \leq A, b \leq B$ for some constants $A, B$, because (C4) is a bound on the product of (squared) axis lengths. Therefore the effective upper bound will be a function of both axis lengths. In particular, for an imbalance factor $\omega \neq 0$, the two lengths will not be able to reach the bound of Eq. 15 simultaneously. The only guarantee is that the value of $4(1+\omega) /\left[\mu^{2}(1-\omega)\right]$ will not be exceeded.

\subsection{Distance from center estimate}

In some scenarios, a good heuristic estimate $p_{c}$ of the paraboloid's planimetric center is available, e.g. the point with the highest $Z$ coordinate within a coniferous tree's point cloud. A mechanism for constraining the fitted paraboloid's center to lie within a predefined distance from $p_{c}$ could therefore be useful. Here, instead of a Euclidean distance constraint we utilize a box variant such that the point $\left(x_{0}, y_{0}\right)$ must lie within the $X Y$ coordinate square centered on $p_{c}$, with side length $2 \delta$ :

$$
\left(\begin{array}{l}
p_{c, x}-\delta \\
p_{c, y}-\delta
\end{array}\right) \leq_{c}\left(\begin{array}{l}
x_{0} \\
y_{0}
\end{array}\right)=\left(-2 A_{1}\right)^{-1} b_{1} \leq_{c}\left(\begin{array}{l}
p_{c, x}+\delta \\
p_{c, y}+\delta
\end{array}\right)
$$

In the above, $\leq_{c}$ refers to componentwise inequality. Unfortunately, $M^{-1} x \leq_{c} z$ does not imply $x \leq_{c} M z$ for general $M, x, z$, therefore each dimension $x, y$ has to be treated separately. To proceed, we derive explicit formulas for $x_{0}, y_{0}$. Let $p_{c}^{ \pm}=p_{c} \pm(\delta, \delta)^{T}$, then inequality 17 is equivalent to:

$$
\left.\begin{array}{l}
p_{c, x}^{-}\left(4 \phi_{0} \phi_{1}-\phi_{2}^{2}\right) \leq \phi_{2} \phi_{4}-2 \phi_{3} \phi_{1} \leq p_{c, x}^{+}\left(4 \phi_{0} \phi_{1}-\phi_{2}^{2}\right) \\
p_{c, y}^{-}\left(4 \phi_{0} \phi_{1}-\phi_{2}^{2}\right) \leq \phi_{2} \phi_{3}-2 \phi_{0} \phi_{4} \leq p_{c, y}^{+}\left(4 \phi_{0} \phi_{1}-\phi_{2}^{2}\right)
\end{array}\right\}
$$

\subsection{Final program formulation}

4.3.1 General case Here, the final quadratic program containing the presented constraints (C1)-(C5) is directly formulated (Eq. 18). It should be noted that due to the bilinear terms of the form $\phi_{k} \phi_{l}$, each of the constraints (C3),(C4),(C5) is non-convex, and hence the matrix representations $Q_{i}$ of these constraints in the canonical QCQP form are not positive semidefinite. This renders the problem NP-hard.

$$
\begin{aligned}
& \min _{\Phi, \Gamma} \sum_{i=1}^{n} W_{i i} \gamma_{i} \\
& \text { s.t. } \left.\begin{array}{r}
D_{i} \Phi^{T}-z_{i} \leq \gamma_{i} \\
-D_{i} \Phi^{T}+z_{i} \leq \gamma_{i}
\end{array}\right\} \forall_{i \in 1, \ldots, n}(\mathrm{C} 1) \\
& \phi_{0}, \phi_{1} \geq 0 \\
& \phi_{2}^{2}-4 \phi_{0} \phi_{1} \leq-\mu^{2}(\mathrm{C} 4) \\
& \left(1-\omega^{2}\right) \phi_{0}^{2}+\left(1-\omega^{2}\right) \phi_{1}^{2}+\phi_{2}^{2}+\left(-2-2 \omega^{2}\right) \phi_{0} \phi_{1} \leq 0 \text { (C3) } \\
& \left.p_{c, x}^{-}\left(4 \phi_{0} \phi_{1}-\phi_{2}^{2}\right)-\phi_{2} \phi_{4}+2 \phi_{3} \phi_{1} \leq 0\right) \\
& \phi_{2} \phi_{4}-2 \phi_{3} \phi_{1}-p_{c, x}^{+}\left(4 \phi_{0} \phi_{1}-\phi_{2}^{2}\right) \leq 0 \\
& \left.p_{c, y}^{-}\left(4 \phi_{0} \phi_{1}-\phi_{2}^{2}\right)-\phi_{2} \phi_{3}+2 \phi_{0} \phi_{4} \leq 0\right\} \\
& \left.\phi_{2} \phi_{3}-2 \phi_{0} \phi_{4}-p_{c, y}^{+}\left(4 \phi_{0} \phi_{1}-\phi_{2}^{2}\right) \leq 0\right)
\end{aligned}
$$

4.3.2 Special case - equal axes The final 4 inequalities in Eq. 18, associated with constraint (C5), introduce many new nonconvex bilinear terms $\phi_{k} \phi_{l}$. This could significantly complicate the search for the optimal solution, because the optimization algorithm must introduce an auxiliary variable for every such term and perform a search over its domain (Belotti et al., 2009). Therefore, we consider a simplification of 
Problem 18 where the axes are constrained to be of equal length. In this case, the rotation angle $\theta$ no longer makes sense, and there is only one axis length $a$, resulting in 4 actual surface parameters $\phi_{0}, \phi_{3}, \phi_{4}, \phi_{5}$, while $\phi_{1}=\phi_{0}$ and $\phi_{2}=0$. In this setting, constraints (C2)-(C4) become redundant, whereas (C5) now reduces to a series of linear inequalities. Plugging $\phi_{2}=0$ into Eq. 9, we see that $x_{0}=-0.5 \phi_{3} / \phi_{0}$, and $y_{0}=-0.5 \phi_{4} / \phi_{0}$. Also, a simple expression for the axis length $a$ exists: $\phi_{0}=-1 / a^{2}$. Therefore, an upper bound $a \leq \sqrt{2 / \mu}$ is easily representable as a bound on $\phi_{0}$ of the form $\phi_{0} \leq-\mu / 2$. Collecting all constraints and variables, we obtain the final linear program:

$$
\begin{aligned}
& \min _{\phi_{0}, \phi_{3}, \phi_{4}, \phi_{5}, \Gamma} \sum_{i=1}^{n} W_{i i} \gamma_{i} \\
& \text { s.t. } \phi_{0}+\frac{\mu}{2} \leq 0 \\
& \left.D_{i}\left[\phi_{0}, \phi_{0}, 0, \phi_{3}, \phi_{4}, \phi_{5}\right]^{T}-z_{i} \leq \gamma_{i}\right\} \\
& \left.-D_{i}\left[\phi_{0}, \phi_{0}, 0, \phi_{3}, \phi_{4}, \phi_{5}\right]^{T}+z_{i} \leq \gamma_{i}\right\} \forall_{i \in 1, \ldots, n}(\mathrm{C} 1) \\
& -2 \phi_{0} p_{c, x}^{-} \leq q_{3} \leq-2 \phi_{0} p_{c, x}^{+} \\
& \left.-2 \phi_{0} p_{c, y}^{-} \leq q_{4} \leq-2 \phi_{0} p_{y, x}^{+}\right\}
\end{aligned}
$$

Note that this linear program is equivalent (in terms of the fitted shape) to the full QCQP with the imbalance coefficient $\omega$ set to 0 , but significantly easier to optimise.

\section{EXPERIMENTS}

\subsection{Material}

Validation of our method was performed on two independent datasets from (I) the Bavarian Forest National Park in Germany $\left(49^{\circ} 3^{\prime} 19^{\prime \prime} \mathrm{N}, 13^{\circ} 12^{\prime} 9^{\prime \prime} \mathrm{E}\right)$ and (II) the northern interior of the Coast Range in western Oregon, USA $\left(45^{\circ} 18^{\prime} 5^{\prime \prime} \mathrm{N}\right.$, $123^{\circ} 22^{\prime} 51^{\prime \prime} \mathrm{W}$ ). The first study area belongs to the mountain mixed forests zone and consists mostly of Norway spruce (Picea abies) and European beech (Fagus sylvatica). The ALS data were acquired using a Riegl LMS-680i scanner in July 2012 with a nominal point density of 30 points $/ \mathrm{m}^{2}$. The pulse rate was $266 \mathrm{kHz}$. The flying altitude of $650 \mathrm{~m}$ resulted in a footprint size of $32 \mathrm{~cm}$. A total of 18 circular plots of size $500 \mathrm{~m}^{2}$ each were extracted from the point clouds, matching the forest locations which were surveyed in a field inventory campaign. Dataset (II) is characterized by pure silvicultural stands of Douglas-fir (Pseudotsuga menziesii) with vine maple (Acer circinatum Pursh) present in the understory. The acquisition took place in April 2011 with a Leica ALS60 scanner, resulting in a mean point density of ca. 10 points $/ \mathrm{m}^{2}$. Here, a single plot of dimensions $76 \times 121 \mathrm{~m}^{2}$ was scanned. One of the circular plots from (I) and part of the plot from (II) are depicted in Fig. 2.

\subsection{Reference data}

In both datasets, tree segmentation using the method by Reitberger et al. (2009) was performed, obtaining point clusters representing single trees. For Dataset (I), 110 reference tree positions were available from field inventory. For (II), no field data for tree stem positions was available, therefore we have to mark the crown centers as tree positions directly within the point clouds of 169 Douglas firs obtained from segmentation (Fig. 2(b)). Moreover, for a significant number of the considered trees, the tree stem positions measured in the field inventory did not exactly align with the tree's center in the corresponding point cloud (see Fig. 3(a)). Part of the reason for this phenomenon can be attributed to the accuracy of the GNSS measurements, however it is likely that the main cause is associated with the potential discrepancy between the tree tops and measured stem positions when the crowns show a degree of asymmetry or the stems are not perfectly vertical. To ensure that the reference positions better reflect the tree top locations, we also manually created them for Dataset I by visual interpretation in point cloud. It should be noted that the clusters in the plot considered for further processing could have resulted in the introduction of a few clusters with segmentation errors, such as possessing parts of adjacent trees (undersegmentation, see Fig. 3(b)) or representing only a part of a tree (oversegmentation). It could even impose further challenges for tree localization task in the experiment. Therefore, three variations of experimental cases were investigated here for the assessment of tree localization: 1. Dataset (I) based on tree stem positions from field data; 2 . Dataset (I) based on manually adjusted tree centers; 3. Dataset (II) based on manually adjusted tree centers. Additionally, the properties of the reference trees for both datasets, including the average height, number of points per tree and radius of the minimum circle containing the crown, are summarized in Table 1 .

\begin{tabular}{lrr}
\hline Property & Dataset (I) & Dataset (II) \\
\hline Tree species & Norway spruce & Douglas fir \\
Num. trees & 110 & 169 \\
$\begin{array}{l}\text { Avg. height [m] } \\
\text { Avg. enclosing circle }\end{array}$ & 17 & 24 \\
$\quad$ radius [m] & 3.10 & 4.56 \\
$\begin{array}{l}\text { Avg. point density of } \\
\quad \text { crowns [pts } / \mathrm{m}^{2} \text { ] }\end{array}$ & 28.61 & 14.62 \\
\hline
\end{tabular}

Table 1. Properties of reference trees.

\subsection{Experimental setup}

To fit the paraboloid to an individual tree point cluster, we first partitioned the points into a planimetric grid of width $d_{\text {grd }}$. Two widths were considered: 40 and $50 \mathrm{~cm}$. We then created a new point cloud with $X Y$ coordinates corresponding to the grid center points and $Z$ coordinates obtained from the highest original laser point within the corresponding grid cell. This way, the paraboloid was fitted only to the points lying on the surface of the tree, as opposed to points lying within the tree. Empty grid cells were given a weight $W_{i i}$ (see Eq. 3) of 0, otherwise the weight was 1. We conducted a series of experiments, varying the method's key parameters to investigate the quality of tree positions obtained through the proposed paraboloid fitting procedure. In all experiments, we used the median absolute deviation (on the respective dataset) between the reference positions and the planimetric centers of the fitted paraboloids in the role of the error measure. To carry out the optimization of Problem 18, we used the freely available solver package SCIP (Achterberg, 2009). The linear program from Eq. 19 was solved using the software package GLPK (gnu.org, 2013). For the two grid sizes, results varied only by $1-2 \mathrm{~cm}$, therefore only the best result for each experiment is presented $\left(d_{\text {grd }}=50 \mathrm{~cm}\right.$ for Dataset $(\mathrm{I})$ with original reference data, $40 \mathrm{~cm}$ otherwise). The constant $\mu$ in (C4) was set to a value such that the maximum axis length did not exceed $3 \mathrm{~m}$. 


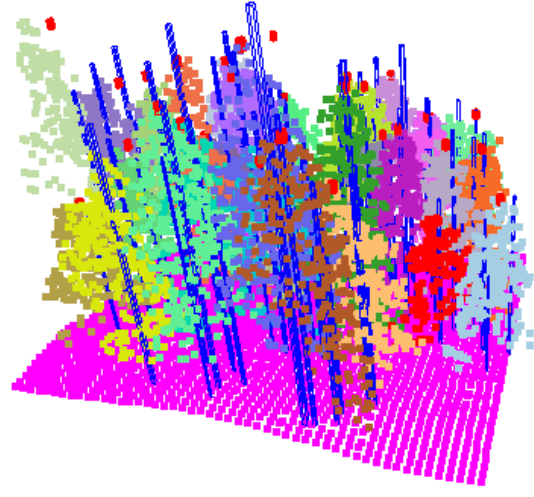

(a)

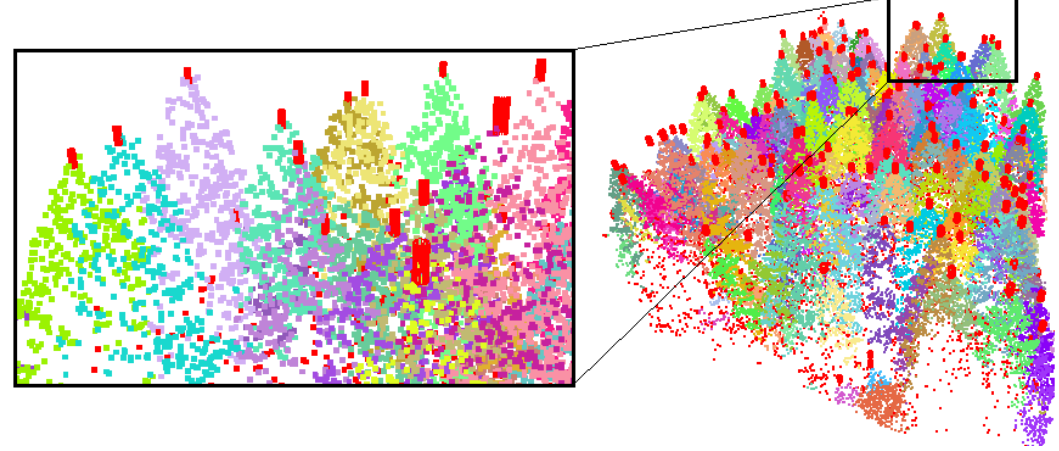

(b)

Figure 2. (a) Point cloud of a circular plot from Dataset (I), with marked tree positions from field inventory (blue bars) and manually refined tree top locations (red cylinders). (b) Manually derived tree positions marked within the point cloud of Dataset (II) by red cylinders. Point colors represent single tree clusters.

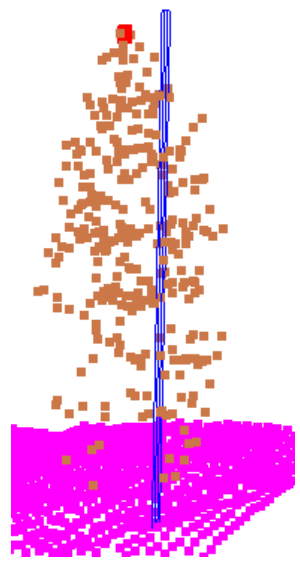

(a)

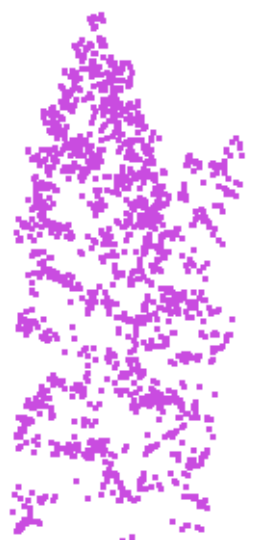

(b)
Figure 3. (a) Discrepancy between tree position from field inventory (blue bar) and true tree top (red cylinder) in Dataset (I). (b) Erroneous tree cluster from Dataset (II), containing part of adjacent tree.

5.3.1 Comparison with baseline methods Here, we compared the positioning errors of our method at varying axis length imbalance coefficient $\omega$ values to errors obtained from competing methods: (i) the centroid of the point cluster's 2D convex hull, (ii) the planimetric position of the highest point within the cluster, and (iii) two least-squares fitting models (Eq. 5) using respectively one and two free axis lengths. The position prior was not included in this experiment (i.e. $\delta=$ inf).

5.3.2 Influence of position prior $\mathrm{We}$ investigated the influence of the parameter $\delta$ quantifying the maximum per-dimension distance to the prior position on the tree position accuracy. The imbalance factor $\omega$ was set to 0 for this experiment. The prior position was derived from the point within the tree's cluster having the highest $Z$ coordinate. Since $\omega$ was 0 , the linear programming formulation of Eq. 19 was applied due to faster solving times.

\section{RESULTS AND DISCUSSION}

\subsection{Comparison with baseline methods}

Figures 4(a)-(c) present the performance of the proposed L1-norm paraboloid fitting approach as a function of the imbalance coefficient, alongside other baseline methods. First, a counter-intuitive result may be seen when comparing the quality of center estimates obtained from the highest point baseline, where the position error is $10 \mathrm{~cm}$ greater on Dataset (I) despite its higher average point density of $28 \mathrm{pts} / \mathrm{m}^{2}$ compared to Dataset (II)'s $14 \mathrm{pts} / \mathrm{m}^{2}$. We believe this may be attributed to the different tree species compositions of these test plots, with spruce trees possibly having a more dense structure in the vicinity of the tree top, containing more side branches. For Dataset (I), an advantage of the L1-norm fitting approach over competing methods can be seen, for both the original field inventory reference and manually marked tree positions. In particular, in the former case an improvement of $13 \mathrm{~cm}$ and $17 \mathrm{~cm}$ is observed compared to respectively the highest point and the centroid methods, while the gain compared to L2 fitting is $1 \mathrm{~cm}$. However, note that these raw reference positions are subject to significant uncertainties (see Fig. 3(a)), resulting in a relatively high median deviation of $58 \mathrm{~cm}$ for the best method. On the other hand, for the manually derived reference tree positions this error can be halved to $0.25 \mathrm{~cm}$. The advantage of L1 fitting over the highest point method still exceeds $10 \mathrm{~cm}$, and nearly reaches $30 \mathrm{~cm}$ compared to the convex hull centroid method's result of $55 \mathrm{~cm}$ (not shown on Fig. 4(b) to maintain scale). Also, this time the improvement w.r.t. L2 fitting is more clear at $4 \mathrm{~cm}$. For Dataset (II), apparently the highest point method already provided a high-quality estimate of the tree position having a median error of $26 \mathrm{~cm}$, with the second-placed method, L1-based fitting, trailing by $13 \mathrm{~cm}$. We believe this is due to the segmentation errors as discussed in Sec. 5.2, resulting in spurious points present within a tree's point cluster (see Fig. 3(b)). It seems that the L1-norm fitting algorithm was more robust to these outliers compared to its L2 counterpart, since the L2 result was worse by a further $13 \mathrm{~cm}$, regardless of whether the model with one or two axis lengths was used. Note that for the raw L2 case, a total of 20 surfaces converged to shapes different than an elliptic paraboloid, which emphasizes the importance of using constraint $(\mathrm{C} 2) /(\mathrm{C} 4)$. Similar to the previous dataset, in (II) the convex hull centroid method also proved to be the weakest, attaining a median error of 124 


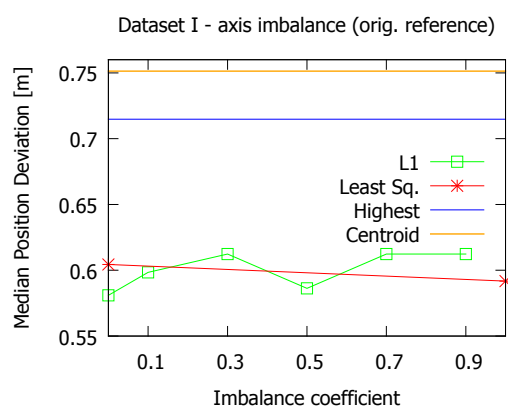

(a)

Dataset I - position prior (orig. reference)

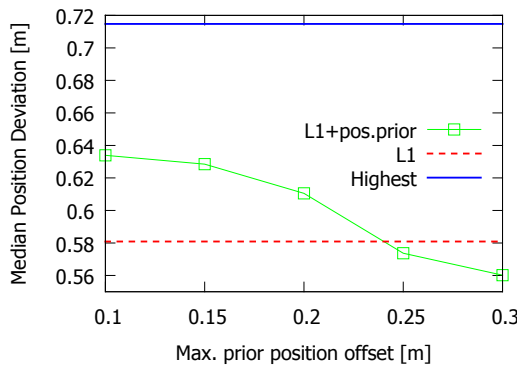

(d)

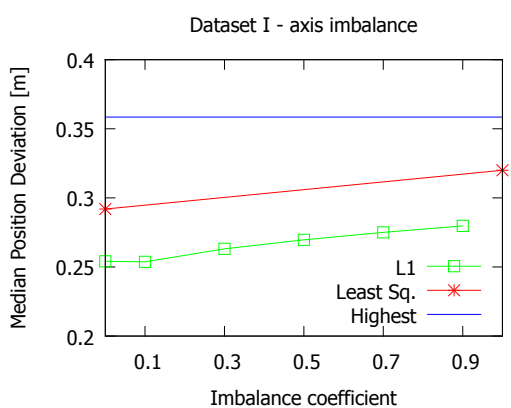

(b)

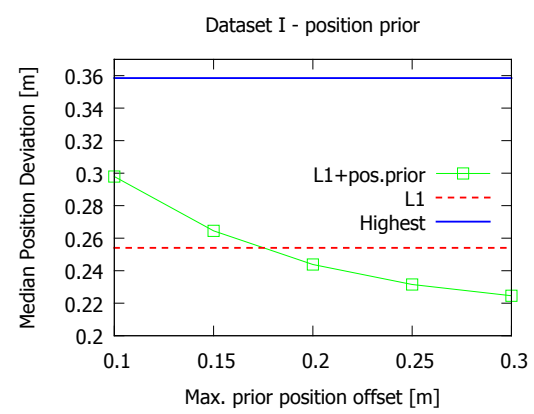

(e)

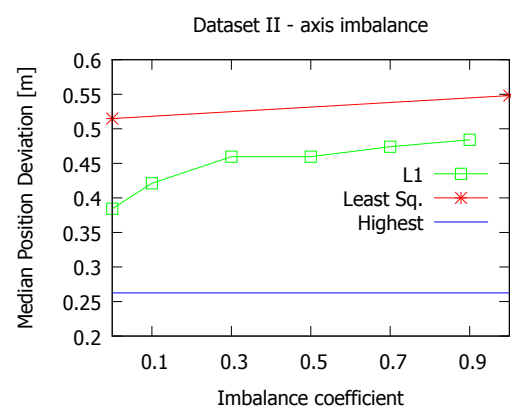

(c)

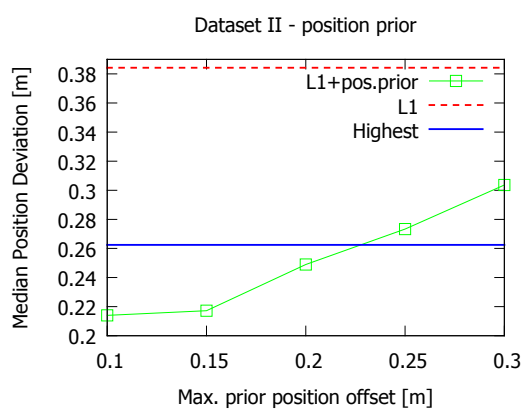

(f)

Figure 4. Median of tree position deviation as a function of maximum allowed axis imbalance (a)-(c) and maximum distance from apriori position (d)-(f). (a),(d) show result of comparison with original, unaltered measurements from field inventory, whereas (b),(c),(e),(f) are based on manually derived tree positions.

$\mathrm{cm}, 400 \%$ worse than the best result. This suggests that the centroid method is not a good estimate of the tree position in the presence of outliers. Perhaps surprisingly, it turned out that allowing a limited amount of imbalance between the paraboloid's semiaxis lengths was not beneficial, and the best results for both datasets were obtained for fully symmetrical models $(\omega=0)$.

\subsection{Influence of position prior}

For both datasets, an increase in accuracy was reached after introducing prior knowledge about the center position (Fig. 4(d)-(f)). Dataset (I) showed gains of $2 \mathrm{~cm}$ and $3 \mathrm{~cm}$ (w.r.t. L1 fitting without the prior) respectively for original and manual reference data at a maximum allowed deviation $\delta=30 \mathrm{~cm}$. Regarding the original reference data case, the final best result was nearly $15 \mathrm{~cm}$ and $20 \mathrm{~cm}$ better compared to respectively the highest point and centroid methods. For (II), a more dramatic gain of $17 \mathrm{~cm}$ vs. L1 fitting without the prior was observed, resulting in a position error of $21.4 \mathrm{~cm}$ for $\delta=10$ $\mathrm{cm}$. This improves the previous lowest error (obtained from the highest point method) by $5 \mathrm{~cm}$. It is not surprising that in case of (II) a relatively small envelope around the highest point leads to the best result, because the highest point is already a good estimate of the tree position. Combined with the geometry information exploited by paraboloid fitting, the prior information-aware method produces a result significantly better than any of the remaining approaches on their own. This synergy effect is particularly important for point clusters containing outliers such as is the case in (II), where it is more difficult to fit a single shape to all such points and the role of an apriori position is emphasized (see Fig. 5). It is interesting to note that although the two datasets were quite different, the best attained accuracy was $21-22 \mathrm{~cm}$ for both of them.

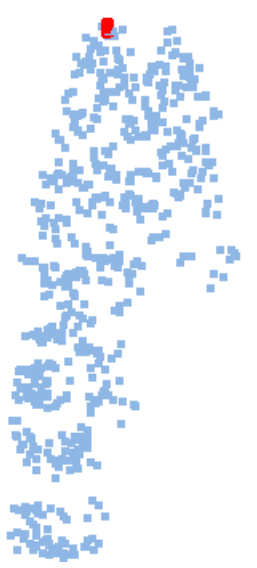

(a)

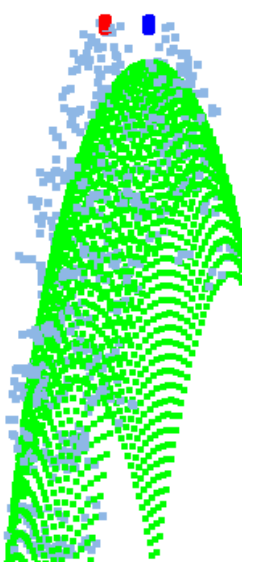

(b)

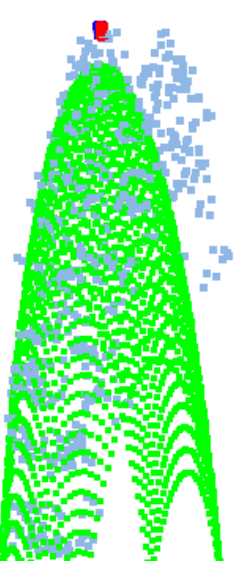

(c)
Figure 5. (a) Undersegmented point cluster with two peaks, reference position marked in red. (b) Best paraboloid fit without position prior, center marked in blue. (c) Best paraboloid fit with position prior, $\delta=10$ $\mathrm{cm}$.

\section{CONCLUSIONS AND OUTLOOK}

We presented a new method for L1-norm fitting of paraboloids with shape and position priors, based on quadratic programming. It was shown that compared to least-squares fitting, the proposed method yields a considerable accuracy improvement of up to $13 \mathrm{~cm}$ in median position accuracy for locating tree positions in $3 \mathrm{D}$ point clusters. Also, the method of calculating the tree position through the centroid of the point cloud's convex hull was outperformed by our approach by up 
to $30 \mathrm{~cm}$. It was found that the centroid is in general not a good estimate of a coniferous tree's center, especially in the presence of outlier points. On the other hand, our results show that the highest point can be a good estimate of the tree position depending on the tree species. Moreover, we showed that by introducing the highest point information into the paraboloid fitting process, the robustness of the method to irrelevant points within the point cluster can be greatly improved, reducing the error by up to $20 \%$. In contrast, the possibility of constraining the imbalance of the paraboloid's semiaxis lengths did not have a significant impact on the surface fitting quality, perhaps due to the inherent symmetry of the investigated tree species (Norway spruce and Douglas fir). In the future, more tight lower bounds could be applied to solve the quadratic problem, for example based on semidefinite or convex quadratic relaxations. It would be interesting to investigate the performance of the proposed method for deciduous trees, although some difficulties could arise due to a less regular shape of tree crowns. Also, our generic formulation of the fitting problem could be re-used in other applications outside of forestry, where asymmetry could play a larger role.

\section{ACKNOWLEDGMENT}

This research was supported in part by a grant PolyU 1-BBWD from the Research Institute for Sustainable Urban Development, the Hong Kong Polytechnic University. The authors would like to thank Adam Erickson for providing the ALS data of the Coast Range plot. Also, we thank Peter Krzystek of the Munich University of Applied Sciences for his support with 3D segmentation of the ALS data.

\section{References}

Achterberg, Tobias, 2009. SCIP: solving constraint integer programs. Mathematical Programming Computation, 1, $1-41$.

Al-Subaihi, I, Watson, GA, 2005. Algebraic fitting of quadric surfaces to data. Communications in Applied Analysis, 9, 539-548.

Amiri, N., Polewski, P., Yao, W., Krzystek, P., Skidmore, A., 2017. Detection of single tree stems in forested areas from high density ALS point clouds using 3D shape descriptors. ISPRS Annals of Photogrammetry, Remote Sensing and Spatial Information Sciences, ISPRS Workshop Laser Scanning.

Amiri, Nina, Polewski, Przemyslaw, Heurich, Marco, Krzystek, Peter, Skidmore, Andrew K., 2018. Adaptive stopping criterion for top-down segmentation of ALS point clouds in temperate coniferous forests. ISPRS Journal of Photogrammetry and Remote Sensing, . Accepted.

Barilotti, A., Crosilla, F., Sepic, F., 2009. Curvature analysis of lidar data for single tree species classification in alpine latitude forests. International Archives of Photogrammetry, Remote Sensing and Spatial Information Sciences, XXXVIII Part 3 / W8.

Belotti, Pietro, Lee, Jon, Liberti, Leo, Margot, Francois, Wächter, Andreas, 2009. Branching and bounds tighteningtechniques for non-convex MINLP. Optimization Methods and Software, 24, 597-634.

Dai, Min, Newman, Timothy S., Cao, Chunguang, 2007. Least-squares-based fitting of paraboloids. Pattern Recognition, 40, 504 - 515.

gnu.org, 2013. GLPK (GNU linear programming kit v.4.52).
Hall, E. L., Tio, J. B. K., McPherson, C. A., Sadjadi, F. A., 1982. Measuring Curved Surfaces for Robot Vision. Computer, 15, 42-54.

Hellström, T., 2008. Autonomous Forest Machines: Past, Present and Future. Department of Computing Science, Umeå University.

Husch, B., Beers, T.W., Kershaw, J.A., 2002. Forest Mensuration. Ecology (John Wiley and Sons), John Wiley \& Sons.

Hussein, Marwan, Iagnemma, Karl, Renner, Matthew, 2015. Global Localization of Autonomous Robots in Forest Environments. Photogrammetric Engineering \& Remote Sensing, 81, 839 - 846.

Lee, Jun-Hak, Biging, Gregory S., Fisher, Joshua B., 2016. An Individual Tree-Based Automated Registration of Aerial Images to Lidar Data in a Forested Area. Photogrammetric Engineering \& Remote Sensing, 82, 699 - 710.

Liang, X., Litkey, P., Hyyppä, J., Kaartinen, H., Vastaranta, M., Holopainen, M., 2012. Automatic Stem Mapping Using Single-Scan Terrestrial Laser Scanning. IEEE Transactions on Geoscience and Remote Sensing, 50, 661-670.

Polewski, Przemyslaw, Yao, Wei, Cao, Lin, Gao, Sha, 2019. Marker-free coregistration of UAV and backpack LiDAR point clouds in forested areas. ISPRS Journal of Photogrammetry and Remote Sensing, 147, 307 - 318.

Pollock, Richard J., 1994. Model-based approach to automatically locating tree crowns in high spatial resolution images.

Reitberger, J., Krzystek, P., Stilla, U., 2007. Combined Tree Segmentation and Stem Detection Using Full Waveform Lidar Data. International Archives of Photogrammetry, Remote Sensing and Spatial Information Sciences, XXXVI Part 3 / W52.

Reitberger, J., Krzystek, P., Stilla, U., 2008. Analysis of full waveform LIDAR data for the classification of deciduous and coniferous trees. International Journal of Remote Sensing, $29,1407-1431$

Reitberger, Joseph, Schnörr, Claudius, Krzystek, Peter, Stilla, Uwe, 2009. 3D segmentation of single trees exploiting full waveform LIDAR data. ISPRS J. Photogramm., 64, 561 574

Xiao, W., Xu, S., Elberink, S. O., Vosselman, G., 2016. Individual Tree Crown Modeling and Change Detection From Airborne Lidar Data. IEEE Journal of Selected Topics in Applied Earth Observations and Remote Sensing, 9, 3467-3477.

Yao, W., Krzystek, P., Heurich, M., 2013. Enhanced detection of 3D individual trees in forested areas using airborne full-waveform LiDAR data by combining normalized cuts with spatial density clustering. ISPRS Annals of Photogrammetry, Remote Sensing and Spatial Information Sciences, II-5/W2, 349-354.

Zhou, L., Salvado, O., 2011. A comparison study of ellipsoid fitting for pose normalization of hippocampal shapes. 2011 International Conference on Digital Image Computing: Techniques and Applications, 285-290. 